\title{
\begin{tabular}{l|l} 
Mibraries & DSpace@MIT
\end{tabular}
}

\author{
MIT Open Access Articles
}

\section{Tunable Raman spectroscopy study of CVD and peapod- derived bundled and individual double-wall carbon nanotubes}

The MIT Faculty has made this article openly available. Please share how this access benefits you. Your story matters.

Citation: Villalpando-Paez, F. et al. “Tunable Raman spectroscopy study of CVD and peapodderived bundled and individual double-wall carbon nanotubes." Physical Review B 82.15 (2010): 155416. (c) 2010 The American Physical Society.

As Published: http://dx.doi.org/10.1103/PhysRevB.82.155416

Publisher: American Physical Society

Persistent URL: http://hdl.handle.net/1721.1/61345

Version: Final published version: final published article, as it appeared in a journal, conference proceedings, or other formally published context

Terms of Use: Article is made available in accordance with the publisher's policy and may be subject to US copyright law. Please refer to the publisher's site for terms of use. 


\title{
Tunable Raman spectroscopy study of CVD and peapod-derived bundled and individual double-wall carbon nanotubes
}

\author{
F. Villalpando-Paez, ${ }^{1}$ L. G. Moura, ${ }^{2}$ C. Fantini, ${ }^{2}$ H. Muramatsu, ${ }^{3}$ T. Hayashi, ${ }^{3}$ Y. A. Kim, ${ }^{3}$ M. Endo, ${ }^{3}$ M. Terrones, ${ }^{4}$ \\ M. A. Pimenta, ${ }^{2}$ and M. S. Dresselhaus ${ }^{5}$ \\ ${ }^{1}$ Department of Materials Science and Engineering, Massachusetts Institute of Technology, Cambridge, Massachusetts 02139-4307, USA \\ ${ }^{2}$ Departamento de Física, Universidade Federal de Minas Gerais, Belo Horizonte, Minas Gerais, Brazil \\ ${ }^{3}$ Faculty of Engineering, Shinshu University, 4-17-1 Wakasato, Nagano-shi 380-8553, Japan \\ ${ }^{4}$ Department of Materials Science and Engineering and Chemical Engineering, Carlos III University of Madrid, \\ Avenida Universidad 30, 28911 Legans, Madrid, Spain \\ and Exotic Nanocarbon Research Center, Shinshu University, 4-17-1 Wakasato, Nagano city 380-8553, Japan \\ ${ }^{5}$ Department of Physics and Department of Electrical Engineering and Computer Science, Massachusetts Institute of Technology, \\ Cambridge, Massachusetts 02139-4307, USA
}

(Received 11 June 2010; revised manuscript received 21 August 2010; published 8 October 2010)

\begin{abstract}
We use 40 laser excitation energies to analyze the differences in the Raman spectra from chemical vapor deposition-derived double-wall carbon nanotube (CVD-DWNT) bundles, fullerene-derived DWNT bundles $\left(\mathrm{C}_{60}\right.$-DWNTs), and individual fullerene-derived DWNTs with inner type-I and type-II semiconducting tubes paired with outer metallic tubes. For the radial breathing mode (RBM) of SWNTs, an experimental $\omega_{\mathrm{RBM}}$ vs $d_{t}$ relationship of the form $\omega_{\mathrm{RBM}}=A / d_{t}+B$ is obtained for the inner tubes of DWNT bundles where the $A$ and $B$ constants are found to be close to those obtained by elasticity theory when modeling the elastic properties of graphite. A similar change in $\omega_{\mathrm{RBM}}$ is observed for an inner type-II semiconducting $(6,5)$ tube and a type-I $(9,1)$ tube when inside various metallic outer tubes. The G-band frequency is observed to upshift when switching the laser resonance from DWNTs with semiconducting inner tubes to DWNTs with metallic inner tubes. Finally, we measure the $\mathrm{G}^{\prime}$ feature from $\mathrm{C}_{60}$-DWNTs and note a downshift in frequency with respect to that of CVD-DWNTs.
\end{abstract}

DOI: 10.1103/PhysRevB.82.155416

PACS number(s): 63.22.- m, 63.22.Gh, 81.07.De

\section{INTRODUCTION}

Double-wall carbon nanotubes (DWNTs) are of scientific interest because they provide the simplest system in which to study the interaction between two concentric carbon nanotubes. Furthermore, the inner and outer tubes of a DWNT can be either metallic $(\mathrm{M})$ or semiconducting $(\mathrm{S})$ so that four different configurations of DWNTs are possible (M@M, S@S, M@S, and S@M). The electronic and optical properties of a DWNT are dependent on the individual properties of its constituent inner and outer tubes and on intertube interactions. Since different DWNT synthesis processes yield different inner-outer tube pairs, the electronic and optical properties of current DWNT samples are highly dependent on the fabrication procedure. The two main fabrication procedures used to synthesize DWNTs include the following: (a) chemical vapor deposition (CVD) (DWNTs) ${ }^{1}$ or (b) the filling of SWNTs with fullerenes where a second inner tube is created by heat-induced coalescence of the fullerenes ( $\mathrm{C}_{60}$-DWNTs). ${ }^{2,3}$ The CVD-DWNTs and $\mathrm{C}_{60}$-DWNTs have been reported to have some differences in their electronic and optical properties due to differences in the crystallinity of the resulting inner tubes and in the characteristic distance between the inner and outer tubes. ${ }^{4}$ For instance, $\mathrm{C}_{60}$-DWNTs have been observed to have smaller average wall to wall distances (defined as the distance between the inner and outer walls of the DWNT) than CVD-DWNTs for DWNTs with similar inner diameters. Also, the photoluminescence (PL) emission from the inner tubes of DWNTs appears to be highly dependent on sample fabrication and pu- rity because both high and low intensity PL signals from the inner tubes of DWNTs have been reported for CVD-DWNTs ${ }^{5}$ and $\mathrm{C}_{60}$-DWNTs, ${ }^{6}$ respectively. The fact that current DWNT synthesis procedures yield samples that contain a mixture of DWNTs with all four of the configurations listed above further complicates Raman data analysis because the Raman spectra from DWNT bundles contain contributions from most of the four possible DWNT configurations. Thus, it is difficult to quantitatively determine the actual $(n, m)$ indices of the constitutive inner-outer tube pairs present in a DWNT bundle. In an effort to study DWNTs with a known configuration, we have performed tunable Raman experiments on DWNT bundles and complemented these studies with careful studies at the individual DWNT nanotube level capable of finding Raman resonances with both the inner and outer tubes of the same DWNT. Herein, we analyze the differences between the Raman spectra obtained from CVD-derived and $\mathrm{C}_{60}$-derived DWNT bundles with 40 laser excitation energies. We also compare the radial breathing mode (RBM) features from individual DWNTs with the RBM features from DWNT bundles.

\section{EXPERIMENTAL DETAILS}

We used a CVD method to synthesize the CVD-DWNT bundles used in this study. ${ }^{1}$ In order to remove unwanted catalyst residues, the sample was heat treated in an $\mathrm{HCl}$ solution at $100{ }^{\circ} \mathrm{C}$ for $10 \mathrm{~h}$. Also, since DWNTs can withstand higher heat treatment temperatures than SWNTs, oxidation in air at $500{ }^{\circ} \mathrm{C}$ for $30 \mathrm{~min}$ was used to remove amorphous 
carbon and impurities in the form of SWNTs. The high purity of our CVD-DWNT bundles relative to residual catalyst particles was confirmed by diamagnetic susceptibility measurements, ${ }^{7}$ and the purity with regard to the absence of SWNTs was confirmed by transmission electron microscopy studies. ${ }^{8,9}$ The $\mathrm{C}_{60}$-DWNT bundles used herein were fabricated by reacting $\mathrm{C}_{60}$ and SWNTs under vacuum conditions at $600{ }^{\circ} \mathrm{C}$ for $24 \mathrm{~h}$. The starting SWNT material was synthesized by the arc method and purified by Hanwha Corporation (Korea). The as-grown peapods (SWNTs containing encapsulated $\mathrm{C}_{60}$ fullerenes) were washed with toluene to remove residual $\mathrm{C}_{60}$ and heat treated at $1700{ }^{\circ} \mathrm{C}$ in $\mathrm{Ar}(1 \mathrm{~atm})$ to transform them into $\mathrm{C}_{60}-\mathrm{DWNT}$ bundles. The individual $\mathrm{C}_{60}$-DWNTs were obtained by dispersing $1 \mathrm{mg}$ of $\mathrm{C}_{60}$-DWNTs in $\mathrm{D}_{2} \mathrm{O}$ with 0.5 wt $\%$ sodium dodecilbenzenesulfonate and sonicating the dispersion $\left(600 \mathrm{~W} \mathrm{~cm}^{-2}\right)$ for 15-30 min. The solution was then ultracentrifuged $(320000 \mathrm{~g}$ ) for $30 \mathrm{~min}$, and $70 \%$ of the supernatant solution was collected. The supernatant solution containing the isolated $\mathrm{C}_{60}$-DWNTs was spin coated on a $\mathrm{Si}$ chip with gold markers, and a Raman mapping technique described elsewhere ${ }^{10}$ was used to obtain Raman resonance spectra from the inner and outer layers of the same individual DWNT. For bundles, the laser power levels were kept below $0.5 \mathrm{~mW}$ to avoid excessive heating of the DWNTs. The scattered light was collected through a $100 \times$ objective using a backscattering geometry. A Nd:YAG laser was used to generate $E_{\text {laser }}=2.33 \mathrm{eV}$ light and a $\mathrm{Kr}^{+}$ion laser generated $E_{\text {laser }}=1.92 \mathrm{eV}$. An argon laser working in the multimode configuration $(6 \mathrm{~W})$ was used to pump a dye laser containing 4-dicyanomethylene-2-methyl-6-p-dimethylamino-styryl$4 \mathrm{H}$-pyran, rhodamine $6 \mathrm{G}$, and $\mathrm{R} 560$ dyes to generate a range of laser energies $E_{\text {laser }}=1.89-1.99 \mathrm{eV}, \quad E_{\text {laser }}$ $=2.05-2.09 \mathrm{eV}$, and $E_{\text {laser }}=2.24-2.30 \mathrm{eV}$, respectively. To generate $E_{\text {laser }}=1.58-1.66 \mathrm{eV}$, a Ti:sapphire laser was pumped by the argon laser. A thermoelectrically cooled $\mathrm{Si}$ charge coupled device detector operating at $-75{ }^{\circ} \mathrm{C}$ was used to collect the Raman spectra.

\section{RESULTS}

\section{A. RBM from CVD-DWNT and $\mathrm{C}_{60}$-DWNT bundles}

We scanned both our CVD-DWNT and $\mathrm{C}_{60}$-DWNT bundled samples with 40 different laser energies $\left(E_{\text {laser }}\right)$ ranging from 1.58 to $2.33 \mathrm{eV}$ and we measured the $\omega_{\mathrm{RBM}}$ values of each identifiable $(n, m)$ tube. The RBM contour maps (two-dimensional color plots of the RBM intensity for $\omega_{\text {RBM }}$ vs $\left.E_{\text {laser }}\right)$ shown in Fig. 1 correspond to (a) CVDDWNTs and (b) $\mathrm{C}_{60}$-DWNTs, and both are superimposed on a SWNT-based Kataura plot of optical transition energy $E_{i i}$ vs the frequency of the radial breathing mode. ${ }^{11}$ The Kataura plot was calculated within the extended tight binding framework, ${ }^{11}$ including many-body corrections, and fitted to the resonance Raman scattering data from sodium dodecyl sulfate wrapped high pressure CO conversion SWNTs. ${ }^{12}$ A SWNT-based Kataura plot was found to be accurate enough for a qualitative identification of the $(n, m)$ indices of the inner and outer tubes of the DWNTs. In principle, each of the four possible DWNT configurations is expected to have (a)

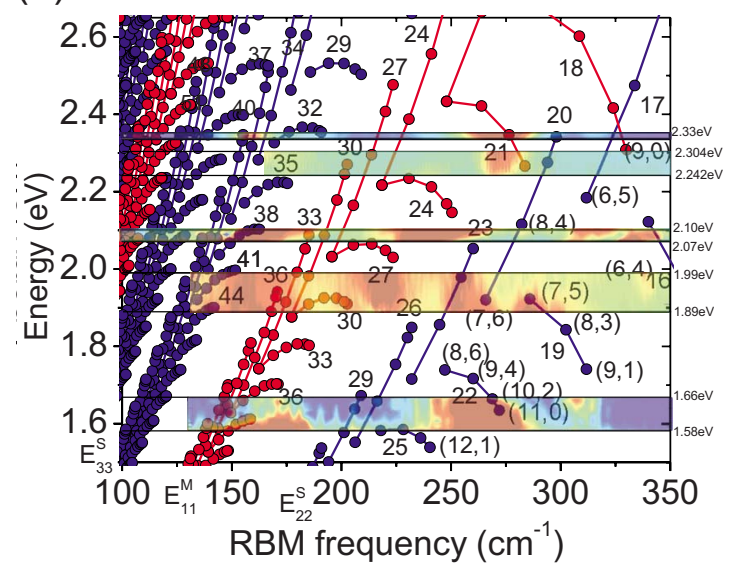

(b)

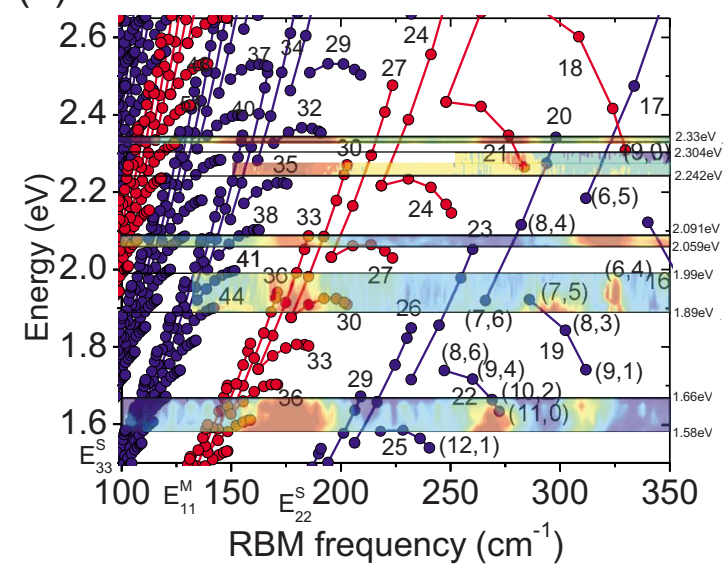

FIG. 1. (Color online) Experimental contour Raman maps of the RBM region from (a) CVD-DWNTs and (b) $\mathrm{C}_{60}$-DWNTs superimposed on a Kataura plot of the resonant transition energies vs RBM frequencies for SWNTs based on the extended tight binding model (Ref. 11). The horizontal lines mark the $E_{\text {laser }}$ regions where Raman spectra were acquired in $5 \mathrm{~nm}$ intervals. In the contour Raman maps the red (blue) color corresponds to regions of maximum (minimum) Raman intensity in arbitrary units.

unique electronic and optical properties and hence to produce a unique Kataura plot. By comparing the Raman maps from CVD-DWNTs and $\mathrm{C}_{60^{-}}$DWNTs in Fig. 1, we find, in agreement with previous work ${ }^{4}$ a marked difference between the diameter distributions of the tubes contained in each type of sample. On one hand, the CVD-DWNT Raman map reveals the presence of inner and outer tubes whose RBMs span the entire range from 140 to $350 \mathrm{~cm}^{-1}$. On the other hand, the $\mathrm{C}_{60}$-DWNT RBM Raman map does not show any resonant tubes in the $190-250 \mathrm{~cm}^{-1}$ range but only shows outer tubes with $\omega_{\mathrm{RBM}}<190 \mathrm{~cm}^{-1}$ and inner tubes with $\omega_{\mathrm{RBM}}>250 \mathrm{~cm}^{-1}$ [see Fig. 1(b)]. This apparent difference in diameter distributions between CVD-DWNT and $\mathrm{C}_{60}$-DWNT bundles arises from the fact that, unlike the CVD-DWNTs, where both inner and outer tubes grow simultaneously, $\mathrm{C}_{60}$-DWNTs experience different diameter constraints during growth. For the case of the $\mathrm{C}_{60}$-DWNTs the possible diameters of the inner tubes are constrained by the diameters of the starting SWNT material $(\sim 1.3-1.4 \mathrm{~nm})$ which forms the outer tubes of the DWNTs. 


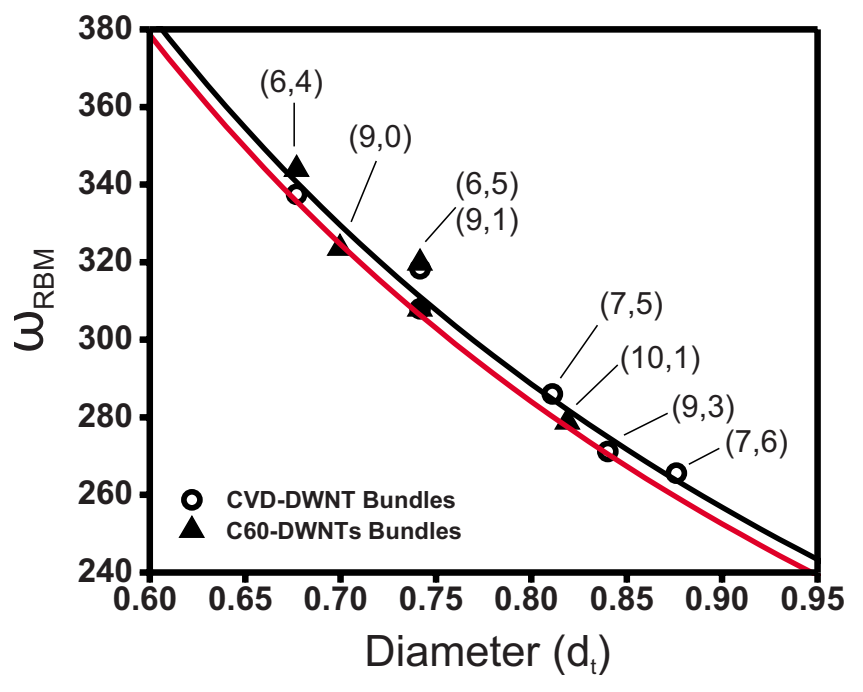

FIG. 2. (Color online) (Top black line) Best fit $\left(\omega_{\mathrm{RBM}}\right.$ $\left.=228.8 / d_{t}+2.4\right)$ to the experimental $\omega_{\mathrm{RBM}}$ vs $d_{t}$ data points from CVD-DWNTs (circles) and $\mathrm{C}_{60}$-DWNTs (triangles). (Bottom red line) Experimental $\omega_{\mathrm{RBM}}$ as a function of $d_{t}$ for supergrowth SWNTs $\left(\omega_{\mathrm{RBM}}=227.0 / d_{t}+0.3\right)$ (Ref. 14).

The relationship between $\omega_{\mathrm{RBM}}$ and $d_{t}$ for the inner tubes and outer tubes of DWNTs is not expected to be the same as it would be for SWNTs with similar diameters. There are no experimental or theoretical $\omega_{\mathrm{RBM}}$ vs $d_{t}$ relationships presently available for the inner and outer tubes that constitute the four different kinds of DWNT configurations $(\mathrm{S} @ \mathrm{~S}$, $\mathrm{S} @ \mathrm{M}, \mathrm{M} @ \mathrm{~S}$, and $\mathrm{M} @ \mathrm{M})$. We thus measured the $\omega_{\mathrm{RBM}}$ values of the identifiable $(n, m)$ tubes in our CVD-derived and $\mathrm{C}_{60}$-derived DWNT samples (see Fig. 2 and Table I) and found the experimental $\omega_{\mathrm{RBM}}$ to $d_{t}$ relationship of the inner tubes to be $\omega_{\mathrm{RBM}}=A / d_{t}+B$, where $A=228.8 \mathrm{~cm}^{-1} \mathrm{~nm}$ and $B=2.4 \mathrm{~cm}^{-1}$. For comparison, in the case of SWNTs, a proportionality constant of $A=227.0 \mathrm{~cm}^{-1} \mathrm{~nm}$ has been reported

TABLE I. The $(n, m)$ index and $d_{t}$ with corresponding experimental $\omega_{\text {RBM }}$ from the inner tubes of CVD-DWNTs (top) and $\mathrm{C}_{60}$-DWNTs (bottom). A horizontal line separates CVD-DWNTs (top) from $\mathrm{C}_{60}$-DWNTs (bottom). The $E_{\text {laser }}$ column denotes the laser energy at which the $\omega_{\text {RBM }}$ was measured (see Fig. 2).

\begin{tabular}{clll}
\hline \hline$(n, m)$ & $\begin{array}{c}d_{t} \\
(\mathrm{~nm})\end{array}$ & $\begin{array}{c}\omega_{\mathrm{RBM}} \\
\left(\mathrm{cm}^{-1}\right)\end{array}$ & $\begin{array}{c}E_{\text {laser }} \\
(\mathrm{eV})\end{array}$ \\
\hline$(6,4)$ & 0.67 & 337.4 & 2.080 \\
$(6,5)$ & 0.74 & 318.3 & 2.100 \\
$(7,5)$ & 0.81 & 286 & 1.898 \\
$(7,6)$ & 0.87 & 265.6 & 1.898 \\
$(9,1)$ & 0.74 & 308 & 1.668 \\
$(9,3)$ & 0.84 & 271.2 & 2.304 \\
\hline$(6,4)$ & 0.67 & 343.9 & 2.059 \\
$(6,5)$ & 0.74 & 319.7 & 2.059 \\
$(9,0)$ & 0.69 & 323.6 & 2.333 \\
$(9,1)$ & 0.74 & 307.9 & 1.668 \\
$(10,1)$ & 0.81 & 278.8 & 2.304 \\
\hline \hline
\end{tabular}

to be the fundamental relation for pristine SWNTs in agreement with the elastic properties of graphite $^{13}$ and $B$ $=0 \mathrm{~cm}^{-1}$ is expected for an ideal case where the effects of the medium surrounding the nanotubes are absent (see Fig. 2 ). The $\omega_{\mathrm{RBM}}$ data for our DWNTs show a slight upshift from the fundamental $\omega_{\mathrm{RBM}}=227.0 / d_{t}$ relation for pristine SWNTs. A list of the small diameter tubes $\left(d_{t}<0.9 \mathrm{~nm}\right)$ whose $(n, m)$ indices were unambiguously identified in the CVD-DWNT and $\mathrm{C}_{60}$-DWNT samples is presented in Table I.

Further inspection of our DWNT bundle spectra reveals a splitting of the RBM from the inner tubes. The RBM splitting in DWNTs has been reported to occur when various inner tubes with a specific $(n, m)$ are contained inside different outer tubes with various diameters and chiralities. When, for a given $(n, m)$ inner tube, the diameter of the outer tube is decreased, the wall to wall distance decreases and the $\omega_{\mathrm{RBM}}$ of the inner tubes upshifts. ${ }^{15-18}$ Thus, a group of DWNTs, with the same $(n, m)$ inner tube and different outer tubes, generates a cluster of RBM peaks and we refer to this effect above as a splitting of its $\omega_{\mathrm{RBM}}$.

In accordance with previous reports, ${ }^{15}$ we observe this RBM splitting effect to be more pronounced in the $\mathrm{C}_{60}$-DWNTs than in the CVD-DWNTs. For instance, the cluster of RBM peaks generated by the $(7,5)$ inner tubes (see Fig. 3) in the $\mathrm{C}_{60}$-DWNT (CVD-DWNT) bundles contains eight (five) distinguishable RBM peaks spread over a 29 (22) $\mathrm{cm}^{-1}$ range. However, we also find that the RBM splitting effect is not necessarily always present for the inner tubes of DWNTs. For instance, the RBMs from the $(6,4)$ inner tubes in $\mathrm{C}_{60}$-DWNT bundles have been reported to span over a $30 \mathrm{~cm}^{-1}$ range, ${ }^{16}$ whereas in our DWNTs the RBMs from the $(6,4)$ inner tubes for both CVD-DWNTs and $\mathrm{C}_{60}$-DWNTs only split within a $<11 \mathrm{~cm}^{-1}$ range [see Figs. 3 (a) and 3(b)]. The small diameter $(7,2)$ inner tube [see Fig. $3(b)]$ observed in the $\mathrm{C}_{60}$-DWNTs also does not show any significant splitting. Thus, even when one would expect smaller diameter inner tubes to show increased splitting because they can fit inside a larger variety of DWNT outer tubes, energy minimizing processes that occur during sample preparation are key to defining the final $(n, m)$ indices and wall to wall distances of the inner and outer tubes that constitute the DWNTs contained in a given sample.

Interestingly, the $(7,2)$ inner tube does not appear in our CVD-DWNT sample [see Fig. 3(a)]. Figure 3 also shows that the inner tubes of the CVD-DWNTs have RBM peaks with larger full width at half maxima (FWHMs) than the $\mathrm{C}_{60}$-DWNTs. This observation suggests that the phonon lifetimes in the inner tubes are longer in $\mathrm{C}_{60}$-DWNTs than in the CVD-DWNTs. However, the intensity of the $\mathrm{D}$ band in $\mathrm{C}_{60}$-DWNTs (not shown here) is greater than that in the CVD-DWNTs, indicating that the former have a larger defect concentration. In a conventional SWNT sample an increased defect concentration would be expected to give rise to more phonon relaxation pathways, decreased phonon lifetimes, and increased RBM FHWM values. ${ }^{19}$ The fact that $\mathrm{C}_{60}$-DWNTs show both sharper RBM peaks and higher D-band intensities than their CVD counterparts suggests that either (a) the $\mathrm{C}_{60}$-DWNT sample contains traces of unwanted amorphous carbon or (b) the majority of the defects in the 

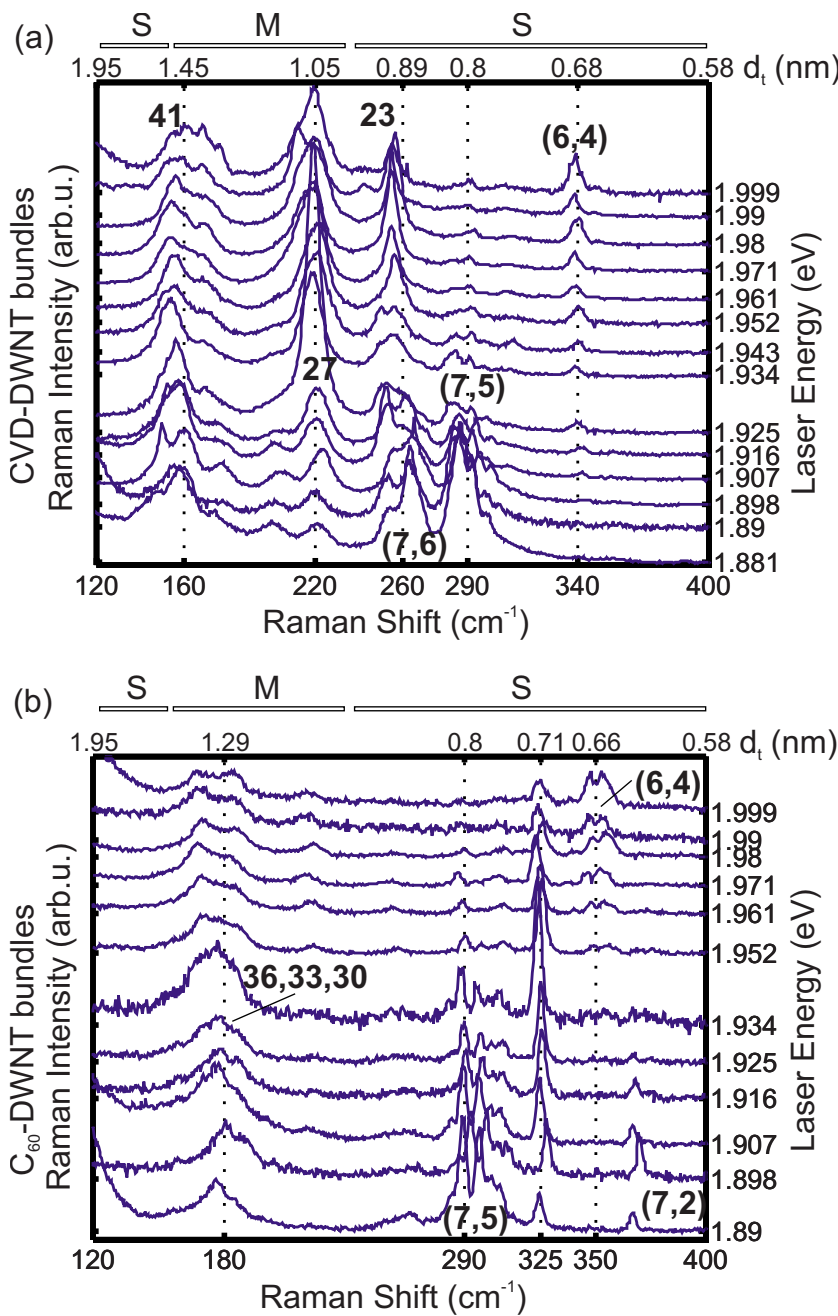

FIG. 3. (Color online) $I_{\text {RBM }}$ vs $\omega_{\text {RBM }}$ for (a) CVD-DWNT and (b) $\mathrm{C}_{60}$-DWNT bundles for the 1.89-1.999 eV $E_{\text {laser }}$ range. The top horizontal axes mark the expected $d_{t}(\mathrm{~nm})$ of the tubes according to our experimentally obtained $\omega_{\mathrm{RBM}}$ vs $d_{t}$ relationship $\left(\omega_{\mathrm{RBM}}\right.$ $\left.=228.8 / d_{t}+2.4 \mathrm{~cm}^{-1}\right)$. The regions marked with an M (S) correspond to metallic (semiconducting) tubes. In the cases where the exact $(n, m)$ of the tube cannot be unambiguously identified, the $(2 n+m)$ family of the corresponding group of tubes is marked.

$\mathrm{C}_{60}$-DWNTs lie in the outer walls which themselves contain crystalline inner tubes that allow for long phonon lifetimes and produce the observed sharp RBM features.

Besides the above-mentioned RBM splitting caused by inner-outer tube interactions in DWNTs, a second mechanism independent of inner-outer tube interactions, known as corrugation of the inner tube, may be capable of generating extra peaks in the RBM region corresponding to the inner tubes of DWNTs. According to Muramatsu et al.,${ }^{20}$ if the fullerenes inside a SWNT are annealed at relatively low temperatures that do not provide the required thermal energy to enable complete fullerene coalescence, the resulting inner tubes will contain nonhexagonal carbon rings and have a corrugated structure. Inner tubes with a corrugated structure may show complex RBM-like vibrational modes. The presence of corrugated inner tubes in our DWNT samples is a possibility but since our sample is heat treated at a high temperature $\left(1700{ }^{\circ} \mathrm{C}\right)$, the so-called corrugation effect is weak and the inner-outer tube interaction is the dominant factor affecting the number and frequency of the observed inner-tube RBMs.

\section{B. RBM from individual $\mathrm{C}_{60}$-DWNTs}

In order to investigate the DWNT configurations contained in our DWNT bundle samples, we isolated DWNTs on a Si substrate and used a Raman mapping technique ${ }^{10,18}$ to obtain Raman resonance spectra from both the inner and outer tubes of the same individual DWNT. The individual DWNT specimens that we specially identified for this study are $\mathrm{C}_{60}-\mathrm{DWNT}$ with an $\mathrm{S} @ \mathrm{M}$ configuration, where the inner $S$ tubes are either $(6,5)$, in resonance with $E_{\text {laser }}$ $=2.10 \mathrm{eV}$, or $(9,1)$, in resonance with $E_{\text {laser }}=1.662 \mathrm{eV}$. Although the $(6,5)$ and $(9,1)$ tubes are both contained inside $\mathrm{M}$ tubes and have almost the same diameter $\left(d_{t}=0.74 \mathrm{~nm}\right)$, the $(6,5)$ tube is a type-II semiconductor and the $(9,1)$ tube is a type-I semiconductor. ${ }^{21}$ Since these semiconducting type-I and type-II tubes have essentially the same diameter but a different chirality dependence of $E_{i i}^{S}{ }^{19}$ we considered that the interaction between each type of inner $\mathrm{S}$ tube with its corresponding outer M tube may vary somewhat. Figure 4 compares the RBM regions of CVD-DWNT bundles and $\mathrm{C}_{60}$-DWNT bundles with the spectra from the individual $\mathrm{S} @ \mathrm{M} C_{60}$-DWNTs whose inner tubes are $(9,1)$ type I and $(6,5)$ type II. As expected, the peaks from the inner tubes of the individual S@M DWNT tubes can be found within the frequency region that is occupied by the broader RBM peak generated by the DWNT bundle samples (see Fig. 4).

In the case of the $(6,5)$ type-II tube, the $\omega_{\mathrm{RBM}}$ from the individual tubes can be found in the $313-329 \mathrm{~cm}^{-1}$ range where the highest RBM frequencies correspond to tubes that are paired with the smallest diameter outer tubes. ${ }^{16,18}$ In the case of the $(9,1)$ inner tubes, the $\omega_{\mathrm{RBM}}$ 's for the two specimens were found to appear in the $311-316 \mathrm{~cm}^{-1}$ range. Similarly, the $\omega_{\mathrm{RBM}}$ 's for the outer tubes pairing with the $(6,5)$ tubes appear in the $175-168 \mathrm{~cm}^{-1}$ range, and the outer tubes pairing with the two $(9,1)$ tubes appear close to $173 \mathrm{~cm}^{-1}$. If we use $\omega_{\mathrm{RBM}}=228.8 / d_{t}+2.4 \mathrm{~cm}^{-1}$ to approximate the wall to wall distance in our individual S@M DWNTs, the specimens with type-I and type-II inner tubes would both have nominal wall to wall distances (in the 0.29$0.32 \mathrm{~nm}$ range) that are 5-15\% smaller than the interlayer spacing in graphite $(0.34 \mathrm{~nm})$. This unexpected result stems from the lack of a $\omega_{\mathrm{RBM}}\left(d_{t}\right)$ relationship capable of taking into account other effects that affect the $\omega_{\mathrm{RBM}}$ such as the $\mathrm{M} / \mathrm{S}$ configuration of the DWNT, the intertube stress on the outer tube, the substrate, and the adsorbed species on the outer tube's wall. Nevertheless, even if we cannot obtain a direct measurement of the actual wall to wall distance, we observe that the $d_{t}$ dependence of the $\omega_{\mathrm{RBM}}$ from the inner and outer tubes of an S@M DWNT is similar to within our experimental error regardless of whether the inner semiconducting tube is type I or type II. We were not able to obtain an $(n, m)$ assignment for the outer tubes from our spectra because the $E_{i i}$ 's from other tubes with similar diameters were too close to each other in energy (see Fig. 1). However, 

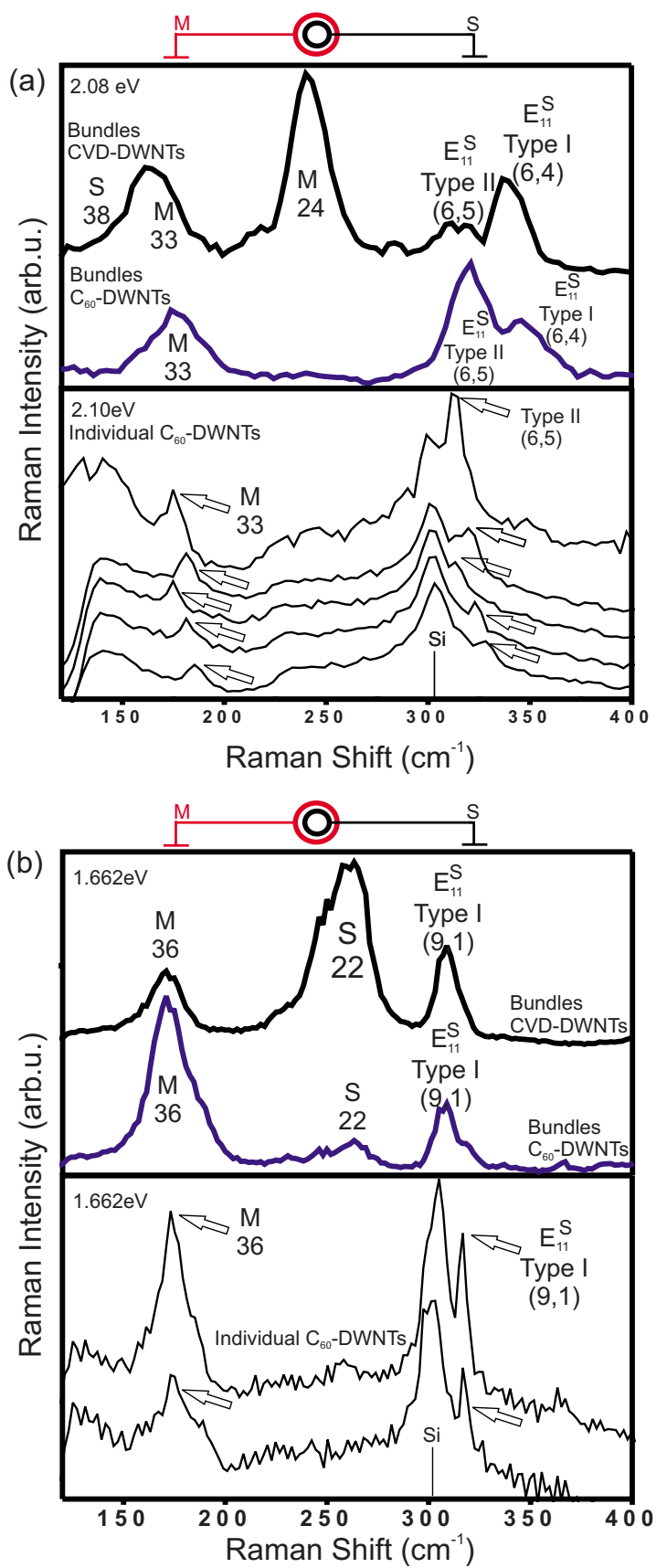

FIG. 4. (Color online) (a) $I_{\mathrm{RBM}}$ vs $\omega_{\mathrm{RBM}}$ from CVD-DWNT bundles, $\mathrm{C}_{60}$-DWNT bundles, and five individual $\mathrm{C}_{60}$-DWNTs (marked by arrows pointing at the inner and outer tube pairs) for similar $E_{\text {laser }}(2.08$ and $2.10 \mathrm{eV})$ excitation energies that excite DWNTs with the S@M configuration. The individual DWNTs have inner tubes that are $(6,5)$ type-II $S$ tubes paired with outer $M$ tubes belonging to $(2 n+m)$ family 33 . (b) $I_{\mathrm{RBM}}$ vs $\omega_{\mathrm{RBM}}$ from CVDDWNTs bundles, $\mathrm{C}_{60}$-DWNT bundles, and two individual $\mathrm{C}_{60}$-DWNTs using $E_{\text {laser }}=1.662 \mathrm{eV}$. The individual S@M DWNTs (marked by arrows pointing at the inner and outer tube pairs) have inner tubes that are $(9,1)$ type-I $S$ tubes paired with outer $M$ tubes belonging to $(2 n+m)$ family 36 .

the data are sufficiently accurate to tell us that the outer tubes of the individual DWNTs with inner $(6,5)$ and $(9,1)$ tubes belong to $(2 n+m)$ families 33 and 36 , respectively.

\section{G band from CVD-DWNT and $\mathrm{C}_{60}$-DWNT bundles}

The $\mathrm{G}$ band is generated by in-plane $\mathrm{C}-\mathrm{C}$ bond stretching modes that occur in the form of optical phonons along the axial [longitudinal optical (LO) mode] and circumferential [transverse optical (TO) mode] directions of a carbon nanotube. ${ }^{19}$ In metallic (semiconducting) tubes, the $\mathrm{G}^{-}$and $\mathrm{G}^{+}$features originate from the LO (TO) and TO (LO) phonons, respectively. ${ }^{22}$ Since the curvature in a nanotube weakens the bonds in the circumferential direction, the $\omega_{\mathrm{G}_{\mathrm{TO}}}$ downshifts with decreasing nanotube diameter.

By measuring the Raman spectra from our DWNTs in the $E_{\text {laser }}=1.58-2.33 \mathrm{eV}$ range (see Fig. 5), we scanned across regions of the resonance windows of the inner and outer tubes that belong to DWNTs with the four possible configurations mentioned above. The inner and outer tubes of the same DWNT are not necessarily in resonance with the same $E_{\text {laser }}$. However, if the RBM and G-band resonance windows of a certain inner tube are centered at roughly the same energy, we can assume, for a certain $E_{\text {laser }}$, that the observed line shape of the $\mathrm{G}$ band is predominantly generated by those tubes that show a resonant RBM signal. We can thus analyze the $\mathrm{RBM}$ region of a spectrum, relate it to its corresponding G-band region, and assume that the tubes with RBMs which are in resonance with a given $E_{\text {laser }}$ have a predominant effect on the observed line shape of the $\mathrm{G}$ band.

In the $E_{\text {laser }}=1.58-1.99 \mathrm{eV}$ range for CVD-DWNTs, we observe that the inner tubes in resonance are predominantly semiconducting (S@S and S@M) [see Fig. 1(a)]. In this case, the frequencies of the $\mathrm{G}^{+}$band $\left(\omega_{\mathrm{G}^{+}}\right)$are centered around $1588 \mathrm{~cm}^{-1}$ with a full width at half maximum intensity of $\mathrm{FWHM}_{\mathrm{G}^{+}} \approx 15 \mathrm{~cm}^{-1}$ [see Fig. 5(a)] and $\omega_{\mathrm{G}^{-}}$ $\approx 1555 \mathrm{~cm}^{-1}$ with $\mathrm{FWHM}_{\mathrm{G}^{-}} \approx 42 \mathrm{~cm}^{-1}$. The presence of the outer $\mathrm{M}$ tubes is likely to be contributing to the large FWHM of $\omega_{\mathrm{G}^{-}}$. On the other hand, for $E_{\text {laser }} \geq 2.07 \mathrm{eV}$, besides being in resonance with S@S and S@M CVD-DWNTs, we also find resonance with CVD-DWNTs having inner metallic tubes (M@S and M@M) that belong to $(2 n+m)$ families 24 and 21 [see Fig. 1(a)] and we observe that the line shape of the $\mathrm{G}$ band undergoes a series of noticeable changes [see Fig. $5(\mathrm{a})]$ as $E_{\text {laser }}$ is increased. These changes in the G-band line shape, which coincide with the appearance of the resonance with $\mathrm{M}$ inner tubes, are (a) an $\omega_{\mathrm{G}^{+}}$upshift of $\sim 7 \mathrm{~cm}^{-1}$ (from $\omega_{\mathrm{G}^{+}} \approx 1588 \mathrm{~cm}^{-1}$ at $1.581 \mathrm{eV}$ to $\omega_{\mathrm{G}^{+}} \approx 1595 \mathrm{~cm}^{-1}$ at 2.33 $\mathrm{eV}$ ), (b) an $\sim 16 \mathrm{~cm}^{-1}$ splitting of the $\mathrm{G}^{+}$band and $\mathrm{G}^{-}$band $\left(\omega_{\mathrm{G}^{-}} \approx 1580 \mathrm{~cm}^{-1}\right.$ and $\omega_{\mathrm{G}^{+}} \approx 1595 \mathrm{~cm}^{-1}$ at $\left.2.33 \mathrm{eV}\right)$, and $(\mathrm{c})$ an $\sim 6 \mathrm{~cm}^{-1}$ increase in FWHM $_{\mathrm{G}^{+}}$(from FWHM $_{\mathrm{G}^{+}}$ $\approx 15 \mathrm{~cm}^{-1}$ at $1.581 \mathrm{eV}$ to FWHM $_{\mathrm{G}^{+}} \approx 21 \mathrm{~cm}^{-1}$ at $\left.2.33 \mathrm{eV}\right)$. The above-mentioned changes can be appreciated in Fig. $5(\mathrm{c})$, where spectra for the two extrema in $E_{\text {laser }}$ are shown (predominant resonance with S@S and S@M DWNTs at $E_{\text {laser }}=1.581 \mathrm{eV}$ to be compared with the resonance with M@S and M@M DWNTs at $E_{\text {laser }}=2.33 \mathrm{eV}$ ).

In the case of $\mathrm{C}_{60}$-DWNTs, the distribution of the four possible DWNT configurations present in the sample is different and we do not find a strong resonance with inner $\mathrm{M}$ tubes belonging to the $(2 n+m)$ family 24 for $E_{\text {laser }}$ =2.242-2.304 eV [see Fig. 5(b)]. Instead we only find strong resonance with inner $M$ tubes for $E_{\text {laser }}=2.33 \mathrm{eV}$ which excites $(9,3)$ inner $M$ tubes belonging to family 21 . 

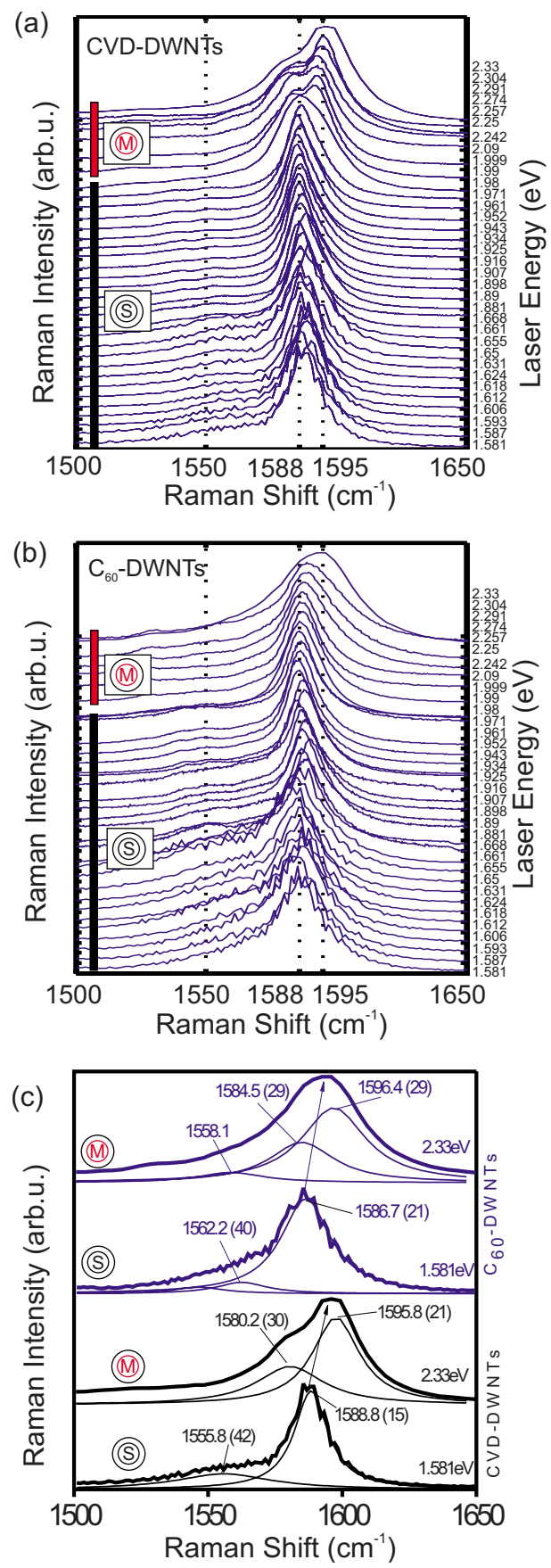

FIG. 5. (Color online) Raman G-band intensity $I_{\mathrm{G}}$ vs $\omega_{\mathrm{G}}$ for the 1.581-2.33 eV $E_{\text {laser }}$ range from (a) CVD-DWNT bundles and (b) $\mathrm{C}_{60}$-DWNT bundles. The dark vertical lines on the left mark the energy regions where the inner tubes in resonance with $E_{\text {laser }}$ are either predominantly semiconducting $\left(E_{\text {laser }}=1.581-1.999 \mathrm{eV}\right.$ resonant with $\mathrm{S} @ \mathrm{~S}$ or $\mathrm{S} @ \mathrm{M})$ or predominantly metallic $\left(E_{\text {laser }}\right.$ =2.09-2.33 eV resonant with $\mathrm{M} @ \mathrm{~S}$ or $\mathrm{M} @ \mathrm{M}$ ). (c) The G-band from $\mathrm{C}_{60}$-DWNT bundles (top) and CVD-DWNT bundles (bottom) for $E_{\text {laser }}$ excitation energies that predominantly resonate with either inner semiconducting tubes $\left(E_{\text {laser }}=1.581 \mathrm{eV}, \mathrm{S} @ \mathrm{~S}\right.$ or $\left.\mathrm{S} @ \mathrm{M}\right)$ or inner metallic tubes $\left(E_{\text {laser }}=2.33 \mathrm{eV}, \mathrm{M} @ \mathrm{~S}\right.$ or M@M).

The line shape of the $\mathrm{G}$ band for $\mathrm{C}_{60}$-DWNTs is similar for all excitation energies in the $E_{\text {laser }}<2.33 \mathrm{eV}$ range where mostly DWNTs with $\mathrm{S}$ inner tubes are excited. However, when $E_{\text {laser }}=2.33 \mathrm{eV}$ is reached and DWNTs with inner M tubes are excited [see Fig. 5(c)], the $\mathrm{G}$ band upshifts $\left(\sim 9 \mathrm{~cm}^{-1}\right.$ increase in $\left.\omega_{\mathrm{G}^{+}}\right)$, splits $\left(\sim 12 \mathrm{~cm}^{-1}\right.$ separation between $\omega_{\mathrm{G}^{-}}$and $\left.\omega_{\mathrm{G}^{+}}\right)$, and broadens $\left(\sim 8 \mathrm{~cm}^{-1}\right.$ increase in FWHM). The upshift and broadening of $\omega_{\mathrm{G}^{+}}$when in resonance with $\mathrm{M} @ \mathrm{~S}$ and $\mathrm{M} @ \mathrm{M}$ DWNTs is consistently observed in both the CVD-DWNTs and $\mathrm{C}_{60}$-DWNTs. Therefore, in DWNT bundles, the line shape of the $\mathrm{G}$ band is not only dependent on the sample's diameter distribution but also on its DWNT M/S configuration distributions.

\section{D. $G^{\prime}$ band from CVD-DWNT and $\mathrm{C}_{60}$-DWNT bundles}

The $\mathrm{G}^{\prime}$ band is based on a two-phonon intervalley double resonance process that does not require the presence of defects. ${ }^{23}$ In general, the $\mathrm{G}^{\prime}$ band is generated by four steps where (a) an incoming photon excites an electron with wave vector $\mathbf{k}$, (b) the electron is scattered from $\mathbf{k}$ to $\mathbf{k}+\mathbf{q}$ by emitting a phonon with wave vector $\mathbf{q}$, (c) the electron is backscattered from $\mathbf{k}+\mathbf{q}$ to $\mathbf{k}$ by emitting a second phonon, and (d) the electron recombines with a hole at k. ${ }^{24}$ Since the laser excitation energy $\left(E_{\text {laser }}\right)$ determines the $\mathbf{k}$ of the excited electron and the $\mathbf{q}$ of the emitted phonons, the $\mathrm{G}^{\prime}$-band mode is dispersive. ${ }^{25}$ For reference, in SWNTs the reported dispersion of the $\mathrm{G}^{\prime}$-band feature is $\sim 106 \mathrm{~cm}^{-1} / \mathrm{eV} .{ }^{19}$ Also, like other Raman modes, the $\mathrm{G}^{\prime}$ band has a diameter dependence.

In DWNT bundles, the diameter distribution is bimodal and we expect a $\mathrm{G}^{\prime}$ band with two main features that correspond to the inner $\left(\mathrm{G}_{1}^{\prime}\right)$ and outer $\left(\mathrm{G}_{2}^{\prime}\right)$ tubes. ${ }^{26}$ The intensity $\left(I_{\mathrm{G}^{\prime}}\right)$, the absolute frequency $\left(\omega_{\mathrm{G}^{\prime}}\right)$, the dispersion $\left(\partial \omega_{\mathrm{G}^{\prime}} / \partial E_{\text {laser }}\right)$, and the FWHM $\left(\mathrm{FWHM}_{\mathrm{G}^{\prime}}\right)$ linewidth of the two $\mathrm{G}^{\prime}$-band components in DWNTs are dependent on nanotube diameter, chirality, metallicity, and other factors, such as doping, strain, and energy of the incoming photons. Figure 6 shows $I_{\mathrm{G}^{\prime}}$ vs $\omega_{\mathrm{G}^{\prime}}$ from (a) CVD-DWNT bundles and (b) $\mathrm{C}_{60}$-DWNT bundles for the $1.881-2.33 \mathrm{eV} E_{\text {laser }}$ range. The CVD-DWNT sample shows a two peaked structure where the low frequency peak $\left(\mathrm{G}_{1}^{\prime}\right)$, which corresponds to the inner tubes, ${ }^{26,27}$ has a higher intensity than its higher frequency counterpart $\left(\mathrm{G}_{2}^{\prime}\right)$. The $I_{\mathrm{G}_{1}^{\prime} / \mathrm{G}_{2}^{\prime}}$ and $\mathrm{FWHM}_{\mathrm{G}_{1}^{\prime} / \mathrm{G}_{2}^{\prime}}$ ratios (not presented here) show a tendency to increase with increasing $E_{\text {laser }}$ Interestingly, the dispersion of the peak corresponding to the inner tubes $\left(\partial \omega_{\mathrm{G}_{1}^{\prime}} / \partial E_{\text {laser }}=122.21 \mathrm{~cm}^{-1} / \mathrm{eV}\right)$ is considerably larger than that of the outer tubes $\left(\partial \omega_{\mathrm{G}_{2}^{\prime}} / \partial E_{\text {laser }}\right.$ $\left.=89.44 \mathrm{~cm}^{-1} / \mathrm{eV}\right)$, indicating that the inner and outer tubes, which vary in diameter and chirality, possess different sets of $E(\mathbf{k})$ dispersion relations with different slopes and thus enable the selection of different $\mathbf{q}$ wave vectors [see Fig. 6(c)]. The inherent difference between the phonon dispersion relations of the inner and outer tubes also contributes to the final $\partial \omega_{\mathrm{G}^{\prime}} / \partial E_{\text {laser }}$ values of the $\mathrm{G}^{\prime} 1$ and $\mathrm{G}^{\prime} 2$ features.

The $\mathrm{G}^{\prime}$-band spectra from the $\mathrm{C}_{60}$-DWNTs also has a two peaked structure where the intensity of $G_{1}^{\prime}$ relative to $G_{2}^{\prime}$ tends to increase with increasing $E_{\text {laser }}$. Interestingly, we observe a slight downshift in the frequency of the $\mathrm{G}^{\prime}$ band of $\mathrm{C}_{60}$-DWNTs with respect to CVD-DWNTs for spectra taken at the same $E_{\text {laser }}$. In particular, the $\omega_{\mathrm{G}_{2}^{\prime}}$ of the $\mathrm{C}_{60}$-DWNTs is on average $\sim 6.5 \mathrm{~cm}^{-1}$ below its $\mathrm{CVD}$-derived counterpart 

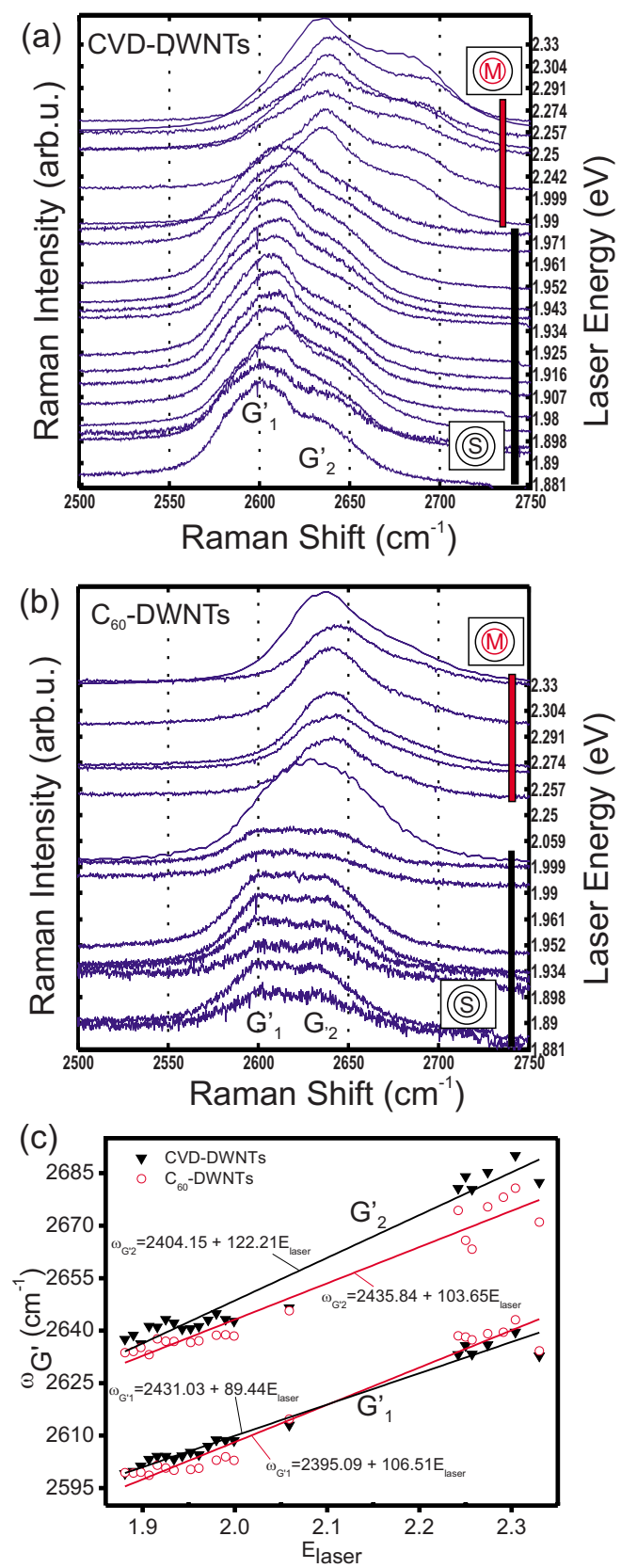

FIG. 6. (Color online) The $I_{\mathrm{G}}$ vs $\omega_{\mathrm{G}^{\prime}}$ from (a) CVD-DWNT bundles and (b) $C_{60}$-DWNT bundles for the 1.881-2.33 eV $E_{\text {laser }}$ range. The dark vertical lines on the right mark the energy regions where the inner tubes in resonance with $E_{\text {laser }}$ are either predominantly semiconducting $\left(E_{\text {laser }}<2.2 \mathrm{eV}\right.$ resonant with $\mathrm{S} @ \mathrm{~S}$ or $\mathrm{S} @ \mathrm{M})$ or predominantly metallic $\left(E_{\text {laser }}>2.2 \mathrm{eV}\right.$ resonant with $\mathrm{M} @ \mathrm{~S}$ or $\mathrm{M} @ \mathrm{M})$. (c) The dispersion of the low $\left(\omega_{\mathrm{G}^{\prime}}\right)$ and high $\left(\omega_{\mathrm{G}_{2}^{\prime}}\right)$ frequency components of the $\mathrm{G}^{\prime}$ band from CVD-DWNT bundles (triangles) and $\mathrm{C}_{60}$-DWNT bundles (circles).

[see Fig. 6(c)]. Similarly, $\omega_{\mathrm{G}_{1}^{\prime}}$ is on average $3.4 \mathrm{~cm}^{-1}$ below its CVD counterpart for $E_{\text {laser }}<2 \mathrm{eV}$. This observation is consistent with the fact that $\omega_{\mathrm{G}^{\prime}}$ scales directly with tube diameter ${ }^{19}$ because the average diameter of the SWNTs that were used to fabricate our $\mathrm{C}_{60}$-DWNTs is likely to be smaller than the outer tube diameters that are present in the CVDDWNT sample. In $\mathrm{C}_{60}$-DWNTs the dispersions of $\mathrm{G}_{1}^{\prime}$ $\left(\partial \omega_{\mathrm{G}_{1}^{\prime}} / \partial E_{\text {laser }}=106.51 \mathrm{~cm}^{-1} / \mathrm{eV}\right) \quad$ and $\quad \mathrm{G}_{2}^{\prime} \quad\left(\partial \omega_{\mathrm{G}_{2}^{\prime}} / \partial E_{\text {laser }}\right.$ $\left.=103.65 \mathrm{~cm}^{-1} / \mathrm{eV}\right)$ are similar and the difference between them $\left(\sim 3 \mathrm{~cm}^{-1} / \mathrm{eV}\right)$ is smaller than the difference between $\partial \omega_{\mathrm{G}_{1}^{\prime}} / \partial E_{\text {laser }}$ and $\partial \omega_{\mathrm{G}_{2}^{\prime}} / \partial E_{\text {laser }}$ observed in the CVD-DWNTs $\left(\sim 30 \mathrm{~cm}^{-1} / \mathrm{eV}\right)$. The fact that the diameter distribution of the inner and outer tubes is narrower in the $\mathrm{C}_{60}$-DWNTs than in the CVD-DWNTs is one of the possible causes for the differences between $\partial \omega_{\mathrm{G}_{1}^{\prime}} / \partial E_{\text {laser }}$ and $\partial \omega_{\mathrm{G}_{2}^{\prime}} / \partial E_{\text {laser }}$ being smaller in $\mathrm{C}_{60}$-DWNTs than in CVD-DWNTs.

From previous experiments performed on SWNTs, the $\omega_{\mathrm{G}^{\prime}}$ was found to have a $d_{t}$ dependence of the form $\omega_{\mathrm{G}^{\prime}}$ $=\omega_{0}+\beta / d_{t}$, where $\omega_{0}=2704 \mathrm{~cm}^{-1}$ and $\beta=-35.4 \mathrm{~cm}^{-1} \mathrm{~nm}$ for $E_{\text {laser }}=2.41 \mathrm{eV} .{ }^{19}$ However, the inner tubes from both CVD-DWNT and $\mathrm{C}_{60}$-DWNT bundled samples show deviations from the above-mentioned SWNT-based $\omega_{\mathrm{G}^{\prime}}\left(d_{t}\right)$ relation. The SWNT-based $\omega_{\mathrm{G}^{\prime}}\left(d_{t}\right)$ relation predicts that our inner tubes with diameters in the $0.7-0.9 \mathrm{~nm}$ range should have $\omega_{\mathrm{G}_{1}^{\prime}}$ in the $2653-2664 \mathrm{~cm}^{-1}$ range. However, for the CVD-DWNT $\left(\mathrm{C}_{60}\right.$-DWNT) sample at $E_{\text {laser }}=2.33 \mathrm{eV}, \omega_{\mathrm{G}_{1}^{\prime}}$ $=2632 \mathrm{~cm}^{-1}\left(\omega_{\mathrm{G}_{1}^{\prime}}=2634 \mathrm{~cm}^{-1}\right)$ which is in both cases below the SWNT-based prediction.

In the case of the outer tubes, there is reasonably good agreement with the SWNT-based $\omega_{\mathrm{G}^{\prime}}\left(d_{t}\right)$ relation. The SWNT-based relation predicts $\omega_{\mathrm{G}^{\prime}}$ to be in the $2676-2681 \mathrm{~cm}^{-1}$ range for tubes with $d_{t}$ in the $1.3-1.6 \mathrm{~nm}$ range and we measure $\omega_{\mathrm{G}_{2}^{\prime}}=2682 \mathrm{~cm}^{-1}\left(\omega_{\mathrm{G}_{2}^{\prime}}=2671 \mathrm{~cm}^{-1}\right)$ for the CVD-DWNTs $\left(\mathrm{C}_{60}\right.$-DWNTs). In agreement with previous reports, ${ }^{26}$ the SWNT-based $\omega_{\mathrm{G}^{\prime}}\left(d_{t}\right)$ relation may predict the $\omega_{\mathrm{G}^{\prime}}$ of the outer tubes quite well but it cannot be applied to the inner tubes because this relation does not take into account the high curvature of tubes with $d_{t}<1 \mathrm{~nm}$ and the possible interactions of the inner tubes with the surrounding outer tubes due to strain and electrostatic effects.

Finally, another factor that affects the lines shape of the $\mathrm{G}^{\prime}$ band is the distribution of the $\mathrm{M} / \mathrm{S}$ configurations present in a DWNT bundle sample. In SWNTs the $\mathrm{G}^{\prime}$-band intensity $\left(I_{\mathrm{G}^{\prime}}\right)$ has been reported to be larger for $\mathrm{M}$ than for $\mathrm{S}$ nanotubes because the electron-phonon matrix elements for the TO phonon at the $K$ point are larger for $\mathrm{M}$ tubes. ${ }^{28}$ Therefore, it is reasonable to expect that the relative intensities of $\mathrm{G}_{1}^{\prime}$ and $\mathrm{G}_{2}^{\prime}$ also depend on whether the inner and outer tubes of a DWNTs are M or S. When taking the metallicity factor into account, one must also keep in mind that the resonance window of the $\mathrm{G}^{\prime}$ band is wider than the resonance window of the RBM and therefore other tubes that are not in resonance with the laser excitation energy for the RBM feature can also contribute to the line shape of the $\mathrm{G}^{\prime}$ band. Resonance with scattered photons can also occur. Nevertheless, if we assume that both the $\mathrm{G}^{\prime}$ band and the RBM resonance windows of a carbon nanotube are centered around the same energy, it is reasonable to expect that the major contribution to the line shape of the $\mathrm{G}^{\prime}$ band in DWNTs comes from the inner and outer tubes that are in resonance with the laser excitation energy. In this context, we divide our $\mathrm{G}^{\prime}$-band measurements into two regimes: (I) for all $E_{\text {lasers }}>2.2 \mathrm{eV}$ that excite M@S DWNTs and the inner M tubes of M@M DWNTs and (II) for all $E_{\text {lasers }}<2.2 \mathrm{eV}$ that excite S@M DWNTs and the inner S tubes of S@S DWNTs (see Figs. 1 and 6). In both 
$\mathrm{C}_{60}$-DWNTs and CVD-DWNTs we observe that the $I_{\mathrm{G}_{1}^{\prime} / \mathrm{G}_{2}^{\prime}}$ is greater in regime II than in regime $\mathrm{I}$ in part because the laser energy is in resonance with the $E_{11}$ transition of the inner M tubes. Thus, when the inner tubes in resonance with $E_{\text {laser }}$ are predominantly metallic, we observe an enhancement of the intensity of the lower frequency $\mathrm{G}_{1}^{\prime}$ region with respect to its high frequency $\mathrm{G}_{2}^{\prime}$ counterpart. Experiments at the individual DWNT level may enable quantitative measurements capable of decoupling the individual contributions to the line shape of the $\mathrm{G}^{\prime}$ band for each of the above-mentioned factors.

\section{CONCLUSIONS}

By scanning a large number of laser energies we have measured the Raman spectra from CVD-derived and $\mathrm{C}_{60}$-derived double-wall carbon nanotubes with the four possible metallic and semiconducting configurations. For small diameter nanotubes $(<0.9 \mathrm{~nm})$, the separation between the $E_{i i}$ is large enough to allow for an accurate $(n, m)$ identification of the inner tubes. Once the $(n, m)$ indices of the observed inner tubes were assigned, we measured their $\omega_{\mathrm{RBM}}$ and obtained an $\omega_{\mathrm{RBM}}$ to $d_{t}$ relationship of the form $\omega_{\mathrm{RBM}}$ $=A / d_{t}+B$. The values of the $A$ and $B$ constants are close to those previously obtained from the elastic properties of graphite, ${ }^{13}$ indicating that the inner tubes are effectively shielded from the environment by their surrounding outer tubes. By scanning the RBM region from the CVD-DWNTs and $\mathrm{C}_{60}$-DWNTs bundles we find that the splitting effect of the $\omega_{\mathrm{RBM}}$ described above is not necessarily always present for all inner tubes. For the same $(n, m)$ inner tubes, the RBM splitting behavior is observed to vary between CVD-derived and $\mathrm{C}_{60}$-derived DWNTs, indicating that the energy minimizing processes inherent to each sample's synthesis method define the final $(n, m) @\left(n^{\prime}, m^{\prime}\right)$ combinations and wall to wall distances found in DWNTs. We also analyzed the Ra- man spectra from individual S@M DWNTs with inner $(6,5)$ type-II and $(9,1)$ type-I semiconducting tubes. The upshift in the $\omega_{\mathrm{RBM}}$ of the inner tube caused by a decrease in the diameter of the outer tube was observed to follow the same trend in both type-I $(6,5)$ and type-II $(9,1)$ inner semiconducting tubes. The line shape and frequency of the $\mathrm{G}$ band from CVD-DWNT and $\mathrm{C}_{60}$-DWNT bundles were analyzed using various laser excitation energies and changes in the G-band line shape were correlated with a switch in resonance for DWNTs whose inner tubes are predominantly semiconducting (S@S and S@M) to resonance with DWNTs with predominantly inner metallic tubes ( $\mathrm{M} @ \mathrm{M}$ and $\mathrm{M} @ \mathrm{~S})$. Finally, the $\mathrm{G}^{\prime}$-band frequency of $\mathrm{C}_{60}$-DWNT bundles is observed to be downshifted with respect to that of CVDDWNTs due to differences in the diameter distributions and $\mathrm{M} / \mathrm{S}$ configuration populations of the samples.

\section{ACKNOWLEDGMENTS}

The authors M.S.D. and F.V.-P. gratefully acknowledge support from NSF/DMR Grant No. 07-04197 and F.V.-P acknowledges financial support from CONACYT graduate student grants, Vilore Foods Co., and SEP Mexico. The authors M.E., Y.A.K., H.M., and T.H. acknowledge support from the Regional Innovation Cluster Program of Nagano, granted by MEXT, Japan, and a grant for Specially Promoted Research (Grant No. 19002007) from the Ministry of Education, Culture, Sports, Science and Technology of Japan. The authors L.G.M., C.F., and M.A.P. acknowledge the support from the Brazilian Network on Nanotube Research and the Brazilian Institute of Science and Technology of Carbon Nanomaterials (CNPq-MCT, Brazil). The authors are grateful to D. Nezich for providing marked Si substrates and to H. Farhat, A. Reina, and M. Hofmann for helpful discussions. The author M.T. acknowledges support from the Japan regional Innovation Strategy Program by the Excellence, JST.
${ }^{1}$ M. Endo, H. Muramatsu, T. Hayashi, Y. A. Kim, M. Terrones, and N. S. Dresselhaus, Nature (London) 433, 476 (2005).

${ }^{2}$ S. Bandow, M. Takizawa, H. Kato, T. Okazaki, H. Shinohara, and S. Iijima, Chem. Phys. Lett. 347, 23 (2001).

${ }^{3}$ S. Bandow, T. Hiraoka, T. Yumura, K. Hirahara, H. Shinohara, and S. Iijima, Chem. Phys. Lett. 384, 320 (2004).

${ }^{4}$ R. Pfeiffer et al., Phys. Status Solidi B 245, 1943 (2008).

${ }^{5}$ D. Shimamoto, H. Muramatsu, T. Hayashi, Y. A. Kim, M. Endo, J. S. Park, R. Saito, M. Terrones, and M. S. Dresselhaus, Appl. Phys. Lett. 94, 083106 (2009).

${ }^{6}$ T. Okazaki, S. Bandow, G. Tamura, Y. Fujita, K. Iakoubovskii, S. Kazaoui, N. Minami, T. Saito, K. Suenaga, and S. Iijima, Phys. Rev. B 74, 153404 (2006).

${ }^{7}$ M. Endo, Y. A. Kim, T. Hayashi, H. Muramatsu, M. Terrones, R. Saito, F. Villalpando-Paez, S. G. Chou, and M. S. Dresselhaus, Small 2, 1031 (2006).

${ }^{8}$ Y. A. Kim, H. Muramatsu, T. Hayashi, M. Endo, M. Terrones, and M. S. Dresselhaus, Chem. Vap. Deposition 12, 327 (2006).

${ }^{9}$ Y. Kim, H. Muramatsu, M. Kojima, T. Hayashi, M. Endo,
M. Terrones, and M. Dresselhaus, Chem. Phys. Lett. 420, 377 (2006).

${ }^{10}$ F. Villalpando-Paez et al., Nano Lett. 8, 3879 (2008).

${ }^{11}$ G. G. Samsonidze, R. Saito, N. Kobayashi, A. Gruneis, J. Jiang, A. Jorio, S. G. Chou, G. Dresselhaus, and M. S. Dresselhaus, Appl. Phys. Lett. 85, 5703 (2004).

${ }^{12}$ A. Jorio, C. Fantini, M. A. Pimenta, R. B. Capaz, G. G. Samsonidze, G. Dresselhaus, M. S. Dresselhaus, J. Jiang, N. Kobayashi, A. Gruneis, and R. Saito, Phys. Rev. B 71, 075401 (2005).

${ }^{13}$ G. D. Mahan, Phys. Rev. B 65, 235402 (2002).

${ }^{14}$ P. Araujo and A. Jorio, Phys. Status Solidi B 245, 2201 (2008).

${ }^{15}$ R. Pfeiffer, C. Kramberger, F. Simon, H. Kuzmany, V. N. Popov, and H. Kataura, Eur. Phys. J. B 42, 345 (2004).

${ }^{16}$ R. Pfeiffer, F. Simon, H. Kuzmany, and V. N. Popov, Phys. Rev. B 72, 161404 (2005).

${ }^{17}$ R. Pfeiffer, F. Simon, H. Kuzmany, V. N. Popov, V. Zolyomi, and J. Kurti, Phys. Status Solidi B 243, 3268 (2006).

${ }^{18}$ F. Villalpando-Paez, H. Muramatsu, Y. A. Kim, H. Farhat, 
M. Endo, M. Terrones, and M. S. Dresselhaus, Nanoscale, 2, 406 (2010).

${ }^{19}$ M. Dresselhaus, G. Dresselhaus, R. Saito, and A. Jorio, Phys. Rep. 409, 47 (2005).

${ }^{20}$ H. Muramatsu, T. Hayashi, Y. A. Kim, D. Shimamoto, M. Endo, V. Meunier, B. G. Sumpter, M. Terrones, and M. S. Dresselhaus, Small 5, 2678 (2009).

${ }^{21}$ M. J. O’Connell et al., Science 297, 593 (2002).

${ }^{22}$ S. Piscanec, M. Lazzeri, J. Robertson, A. C. Ferrari, and F. Mauri, Phys. Rev. B 75, 035427 (2007).

${ }^{23}$ R. Saito, A. Jorio, A. Souza Filho, A. Grueneis, M. A. Pimenta, G. Dresselhaus, and M. Dresselhaus, Physica B 323, 100
(2002).

${ }^{24}$ R. Saito, A. Grüneis, G. G. Samsonidze, V. W. Brar, G. Dresselhaus, M. S. Dresselhaus, A. Jorio, C. Fantini, M. A. Pimenta, and A. G. S. Filho, New J. Phys. 5, 157 (2003).

${ }^{25}$ R. Saito, A. Jorio, A. G. S. Filho, G. Dresselhaus, M. S. Dresselhaus, and M. A. Pimenta, Phys. Rev. Lett. 88, 027401 (2002).

${ }^{26}$ R. Pfeiffer, H. Kuzmany, F. Simon, S. N. Bokova, and E. Obraztsova, Phys. Rev. B 71, 155409 (2005).

${ }^{27}$ E. B. Barros et al., Phys. Rev. B 76, 045425 (2007).

${ }^{28}$ K. K. Kim, J. S. Park, S. Kim, H. Z. Geng, H. K. An, C. Yang, K. Sato, R. Saito, and Y. Lee, Phys. Rev. B 76, 205426 (2007). 